\title{
Nutrient-Dependent Acetylation Controls Basic Regulatory Metabolic Switches and Cellular Reprogramming
}

\author{
J.E. Dominy, Z. Gerhart-Hines, and P. Puigserver \\ Department of Cancer Biology, Dana-Farber Cancer Institute and Department of Cell Biology, \\ Harvard Medical School, Boston, MA 02115 \\ Correspondence: pere_puigserver@dfci.harvard.edu
}

\begin{abstract}
Organisms must be able to selectively tailor their ability to use the macronutrients of carbohydrate, protein, and fat based on their availability. In different cell types, how the nutrient fluctuations are sensed and the mechanisms by which the pathways of central metabolism are switched to favor the use of one particular nutrient type over another are topics of intense interest. Protein acetylation is one major evolutionary conserved mechanism by which nutrient fluctuations are sensed within cells and subsequently coupled with metabolic switching. In this review, we present the case of PGC-1 $\alpha$ acetylation and how the control of PGC-1 $\alpha$ 's activity by acetylation sets into motion a wide range of metabolic adaptations that makes this protein an exemplary model for acetylation-mediated mechanisms of nutrient sensing and communication.
\end{abstract}

In response to the inherently unpredictable nature of nutrient and energy availability, mammalian cells have evolved a complex regulatory system for controlling their rates of mass accretion and energy flux through fuel/ macronutrient utilization pathways. Often, this requires a "metabolic switch" wherein a given cell type is forced to stop using one type of macronutrient as a fuel source and convert to the use of another. Under energy-demanding conditions, for instance, most mammalian cells rapidly initiate a metabolic response that limits glucose oxidation and enhances fatty acid oxidation. This tissue-specific cellular reprogramming is integrated across the whole body and allows for the sparing of glucose for cells that largely depend on this nutrient, such as neurons and red blood cells. Molecular studies of the signal transduction and transcriptional components that are involved in this metabolic reprogramming have revealed that many of these regulatory pathways rely on the PGC family of transcriptional coactivators. PGC- $1 \alpha$, the prototypic member of the PGC family, is able to coordinate the expression of the key enzymes involved in both the breakdown and synthesis of glucose and fatty acids. The nutrientmediated control of PGC- $1 \alpha$ function is tightly coupled with its acetylation state. Herein, we review how cellular nutrient switches and metabolic reprogramming during energetic stresses/demands are regulated by PGC- $1 \alpha$ acetylation. This posttranslational modification axis is ultimately defined by the relative activities of the deacetylase Sirt1 and the acetyltransferase GCN5. These two evolutionary conserved enzymes are under strict control by signaling transduction and metabolic pathways as well as by other components of the transcriptional machinery. Understanding the basic underlying mechanisms of this control will lead to new insights into fundamental cellular energetic processes that are important for the maintenance of metabolic homeostasis in mammals. Moreover, in the context of human disease, these mechanisms will provide the molecular basis for the possible development of new treatments for metabolic disorders such as type II diabetes and obesity.

\section{PROTEIN ACETYLATION: AN EVOLUTIONARILY CONSERVED REGULATORY MECHANISM FOR THE CONTROL OF CENTRAL METABOLISM}

Although in this review we concentrate on the control of metabolic processes by protein acetylation in mammalian cells, changes in protein acetylation constitute a major regulatory theme that is conserved from bacteria to humans. Indeed, the two classes of enzymes that are responsible for the addition and removal of protein lysine acetylation, acetyltransferases and deacetylases, are present in all cellular organisms. Why have cellular systems evolved to use acetylation as a posttranslational modification of proteins to control and adapt to a nutrientmediated response? A simple answer may be found in the fact that most of the central macronutrient metabolic pathways (either anabolic or catabolic) converge on two key molecules: acetyl CoA (a substrate for acetyltransferases) and $\mathrm{NAD}^{+}$(a cosubstrate for a subset of deacetylases called the Sirtuin proteins). Changes or perturbations in central metabolism, more often than not, result in changes in acetyl $\mathrm{CoA}$ and $\mathrm{NAD}^{+}$levels and thus directly alter the activity of acetyltransferases and Sirtuin deacetylases. In this sense, changes in a nutrient/metabolic pathway can be transduced into a signaling and/or transcriptional 
regulatory mechanism that will subsequently affect fundamental cellular processes such as cell division, energy storage or utilization, and survival. In many cases, nutrient-driven changes in protein acetylation affect transcription of the genes directly involved in mediating the flux of central metabolism (such as enzymes and metabolite transporters) through the intermediacy of both histone and nonhistone protein acetylation. Conceptually, this is similar to the transcriptional feedback mechanisms of enzymatic metabolic control described by the bacterial lac operon/repressor model discovered in the last century by Jacob and Monod (Jacob et al. 1960).

\section{SIGNALING TRANSDUCTION CONTROL OF METABOLIC SWITCHES AND CELLULAR REPROGRAMMING}

When a metabolic switch, such as a switch from glucose to fatty acid oxidation, is activated, there is a global remodeling of the pathways of central metabolism that are involved in dictating the macronutrients a cell can use for energy production and biomolecular synthesis. In mammalian cells, the process by which a metabolic switch is triggered is a complex event that involves the integration of many different signals including fluctuations of nutrients, hormones, circadian cycles, chemicals and xenobiotics, and external changes such as temperature, light, and mechanical stress. The integrated contribution of multiple signals to metabolic switching is clearly evidenced in the liver under fasting conditions, wherein decreases in plasma glucose result in a decrease in ChREBP signaling, insulin signaling is attenuated owing to a reduction in circulating insulin levels, and cAMP-mediated signaling is elevated in response to higher levels of blood glucagon and catecholamines. The convergence of these nutrient and hormonal signals in the liver is the key impetus for the metabolic reprogramming of hepatocytes during fasting, yielding changes in the expression of genes involved in glucose production, fatty acid oxidation, and ketone body synthesis, all of which are required to survive periods of starvation.

Before delving into a further discussion about the nutrient and hormonally mediated control of PGC-1 $1 \alpha$ acetylation, we highlight some of the major signaling pathways that are activated by commonly encountered external stimuli. These regulatory cascades serve to initiate the sort of metabolic switches that reprogram cells to use different macronutrients and implement new biomolecular functions, such as cell growth, migration, heat production, contraction, synapsis, and glucose production. Incidentally and not surprisingly, these pathways also influence PGC-1 $\alpha$ acetylation.

\section{cAMP-Protein Kinase A Pathway}

This is an ancient signaling pathway that relies on increases in intracellular cAMP concentrations. Binding of cAMP molecules to the regulatory components of the protein kinase A (PKA) tetrameric complex facilitates the release and activation of catalytic subunits. From a metabolic standpoint, the cAMP pathway is generally associated with the activation of catabolic pathways in response to cellular stresses, for example, the catecholamine-mediated activation of lipolysis of triglyceride stores to produce free fatty acids or the liberation of free glucose from stored glycogen during the fight-or-flight response. Many of the immediate transcriptional changes that accompany activation of the cAMP-PKA axis are mediated through the CREB/ATF transcription factors and its associated accessory factors such as PGC- $1 \alpha$ (Mayr and Montminy 2001). Exposure to low temperatures, for instance, initiates widespread adaptive physiological changes that predominantly depend on a functional cAMP-PKA pathway. One hallmark of this adaptive response is increased expression of thermogenic genes, namely, uncoupled protein-1, that promote uncoupled mitochondrial respiration and the release of heat in brown adipose tissue (Cannon and Nedergaard 2004). Importantly, deletion of PGC- $1 \alpha$ dramatically impairs this response in cold-challenged mice (Lin et al. 2004).

\section{AMPK}

AMP-activated protein kinase (AMPK), by responding to changes in the level of AMP, is effectively tied into the cell's adenylate charge and overall energetic state (Hardie 2007). In general, activation of AMPK begins a cascade of catabolic pathways - such as fatty acid oxidation - for the purposes of yielding additional energy for the cells during times of high energy demand or low substrate availability, such as physical exercise or caloric restriction states. Concurrently, AMPK inhibits anabolic pathways such as glycogen synthesis and lipogenesis through the phosphorylation of key regulatory enzymes such as acetyl-CoA carboxylase, which is involved in de novo fatty acid synthesis (Kahn et al. 2005). Chronic activation of AMPK also activates mitochondrial biogenesis and improves energetic efficiency through direct and indirect control of nuclear transcriptional components. The complete molecular mechanisms are not completely understood. However, among direct AMPK targets are PGC- $1 \alpha$ and SIRT1, known key modulators of mitochondrial function.

\section{PI3K and mTOR Pathways}

Most of the metabolic functions of this anabolic pathway might be considered, at least in general terms, contrary to those of the AMPK and cAMP catabolic pathways described above. Indeed, the PI3K/Akt pathway is considered to be the main mediator of insulin's metabolic actions - such as suppression of fatty acid oxidation and gluconeogenesis, and the activation of glycolysis and fatty acid synthesis - in mammalian tissues. One of the main effectors of AKT is the mTOR kinase pathway, which has been implicated in coordinating cellular proliferation with changes in mitochondrial oxidative phosphorylation capacity. Interestingly, in skeletal muscle, mTOR binds to the transcription factor YY1 and recruits PGC- $1 \alpha$ in a process that facilitates YY1's ability to promote the 
expression of genes involved in mitochondrial proliferation and oxidative phosphorylation (Cunningham et al. 2007).

\section{FGF21}

One of the central metabolic reprogramming switches is the process of energy substrate selection, whereby a cell shifts from glucose to fatty acids as a main carbon source for the generation of ATP. One of the hormones that controls this process is FGF21, which is induced upon fasting conditions and targets peripheral tissues, including skeletal muscle, adipose tissue and liver. Within these tissues, FGF21 potently activates PPAR $\alpha$-dependent mitochondrial fatty acid oxidation and ketone body production (Inagaki et al. 2007; Badman et al. 2009). Interestingly, FGF21 also signals through SIRT1 deacetylase and PGC-1 $\alpha$ to promote the activity of these catabolic pathways (Chau et al. 2010; see below).

\section{TRANSCRIPTIONAL CONTROL OF METABOLIC SWITCHES AND CELLULAR REPROGRAMMING: THE CASE OF THE PGC-1 $\alpha$ PATHWAY}

A metabolic switch can occur in response to a change in nutrient availability or a change in the hormonal milieu. The early response phases of this switch are characterized by changes in metabolic pathway flux brought about through alterations in enzyme/transporter activity that are mediated by posttranslational modifications through signaling cascades, alterations in the balance of substrate and product-mediated effects on enzyme reaction rates, and allosterism. These acute mechanisms of control are often immediately followed by a broader transcriptional response that leads to the regulation of specific metabolic pathways by affecting expression of genes encoding for enzymes and/or other proteins that will aid in the long-term maintenance of the metabolic switch and improve the adaptive capacity of the cell.

As a transcriptional coactivator, it is within this latter phase of metabolic switching that PGC- $1 \alpha$ is recruited by the cell to effect changes in metabolic remodeling. Whereas nuclear transcription factors control specific sets of genes through direct binding to DNA, cell regulatory networks have evolved to use transcriptional coactivators to control metabolic switches across multiple pathways. The PGC family of coactivators (PGC- $1 \alpha$, PGC-1 $\beta$, and PRC) affects a large number of genes involved in oxidative metabolism and mitochondrial function (Puigserver and Spiegelman 2003; Kelly and Scarpulla 2004). In fact, both PGC- $1 \alpha$ and PGC- $1 \beta$, are sufficient to induce mitochondrial biogenesis through an increase in mitochondrial gene expression. Specific mitochondrial genes affected by these coactivators include OXPHOS components, reactive oxygen species scavenging enzymes, mitochondrial-specific metabolic pathways that take place, at least partially, inside the organelle, import proteins, mitochondrial ribosomal proteins, mitochondrial transcription factors, fusion/fission proteins, and mitochondrial sirtuins (Mootha et al. 2003; Cunningham et al. 2007; Rasbach et al. 2010). Factors that control the activity of these transcriptional coactivators thus have the capacity to sculpt flux through many of the pathways of central metabolism. One such factor is acetylation.

\section{PROTEIN ACETYLATION IS AN IMPORTANT REGULATOR OF PGC-1 $\alpha$ ACTIVITY AND IS CONTROLLED BY GCN5 AND Sirt1}

There are at least 13 different lysine acetylation sites that have been identified on PGC- $1 \alpha$ (Rodgers et al. 2005). The major acetyltransferase responsible for PGC$1 \alpha$ acetylation in vivo is GCN5 (KAT2A) (Lerin et al. 2006). The removal of acetylation from PGC- $1 \alpha$ is effectively catalyzed by the Sirtuin protein deacetylase Sirt1 (Rodgers et al. 2005). The relative difference in activities between these two enzymes is an important determinant in the steady-state level of PGC- $1 \alpha$ acetylation and its cotranscriptional activity.

From the standpoint of biological activity, acetylation is strongly associated with a repression of PGC- $1 \alpha$ function, whereas deacetylation is strongly associated with an increase in PGC- $1 \alpha$ function. Hyperacetylation of PGC- $1 \alpha$ by GCN5 causes a redistribution of PGC-1 $\alpha$ 's subnuclear localization, sequestering the protein within punctate nuclear bodies along with its transcription factor binding partners. In this state, the PGC- $1 \alpha$ complex appears to be effectively transcriptionally inactive (Lerin et al. 2006). Deacetylation of PGC- $1 \alpha$ by Sirt1, in contrast, coincides with an increased occupancy of PGC- $1 \alpha$ at the promoters of its target genes (Gerhart-Hines et al. 2007). Additional work is needed to identify how the acetylation state of PGC- $1 \alpha$ is "sensed" by the nuclear machinery responsible for its relocalization as well as the identity of the molecular components needed for this trafficking.

\section{Factors Affecting PGC-1 $\alpha$ Acetylation}

Are there particular nutrient or hormonal states that affect PGC- $1 \alpha$ acetylation and what are the mechanisms by which these changes occur? In general, experiments from both cell culture and whole animal studies have shown that there is a positive correlation between nutrient availability and PGC- $1 \alpha$ acetylation. On the other hand, there is a strong negative correlation between the energy demands of a cell and PGC- $1 \alpha$ acetylation. Biochemical and genetic dissection of these phenomena has begun to reveal that there are multiple signaling pathways that target the activities of GCN5 and Sirt1 and modulate PGC$1 \alpha$ acetylation. We describe the pathways that are known to modulate the activities of GCN5 and Sirt1 below and present a graphical summary of these findings in Figure 1.

\section{The Relationship between GCN5 and PGC-1 $\alpha$ Acetylation and its Regulation by Nutrient Supply and Hormones}

The interaction between GCN5 and PGC-1 $\alpha$ requires the carboxy-terminal bromodomain of GCN5 and amino 


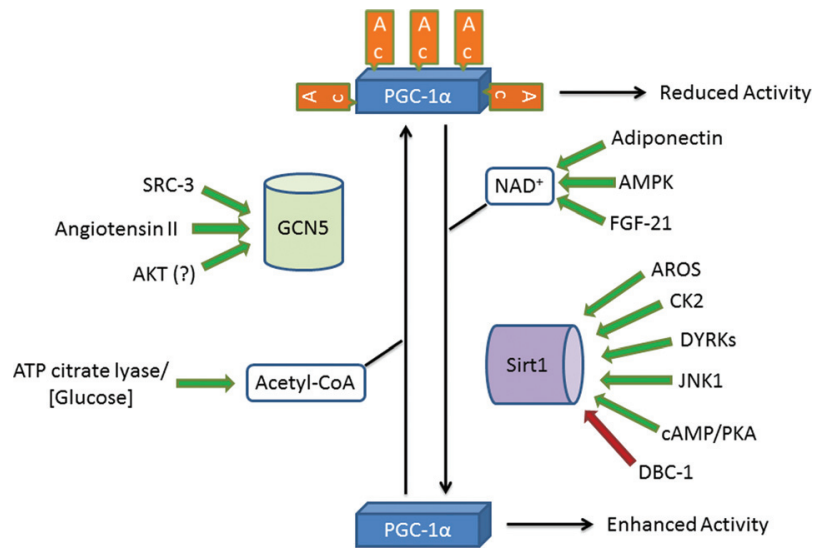

acids $1-200$ of PGC- $1 \alpha$. GCN5's binding to and acetylation of PGC- $1 \alpha$ are associated with a decrease in PGC$1 \alpha$ cotranscriptional activity. Overexpression of GCN5, for example, was able to effectively suppress the induction of PGC- $1 \alpha$ target genes caused by the overexpression of wild-type PGC- $1 \alpha$ in cultured hepatoma cells (Lerin et al. 2006). On the other hand, reduction of GCN5 in these cells enhanced the function of PGC-1 $\alpha$. In mice, the hepatic overexpression of GCN5 during fasting resulted in a suppression of the gluconeogenic genes phosphoenolpyruvate carboxykinase (PEPCK) and glucose-6phosphatase (G-6-Pase) as well as in a reduction of de novo synthesis of glucose from pyruvate (Lerin et al. 2006). In myotubes, overexpression of GCN5 also yielded an increase in PGC- $1 \alpha$ acetylation and produced a significant decrease in the expression of PGC- $1 \alpha$ target genes, such as cytochrome $\mathrm{C}$ and pyruvate dehydrogenase kinase 4 (Gerhart-Hines et al. 2007).

One of the ways in which macronutrient availability could modulate PGC- $1 \alpha$ acetylation through GCN5 is through steroid receptor coactivator protein (SRC-3) (Coste et al. 2008). SRC-3 positively regulates the activity of GCN5 in mammalian cells. SRC-3 has been shown to bind to GCN5 and this interaction is needed for SRC-3 to coactivate retinoic acid receptor (Brown et al. 2003). Knockout of SRC-3 in mice has been reported to significantly reduce the expression of GCN5 in muscle and brown adipose tissue (Coste et al. 2008). Both muscle and brown adipose tissue showed significantly lower levels of PGC-1 $\alpha$ acetylation in SRC-3 knockout mice. Consistent with the change in PGC- $1 \alpha$ acetylation levels, many mitochondrial gene targets of PGC- $1 \alpha$, such as CPT-1, UCPs-1-3, cytochrome C, and ATP synthase, were significantly elevated relative to wild-type controls (Coste et al. 2008). In wild-type mice, both SRC-3 and GCN5 expression positively correlated with nutrient availability. Following a short-term fast $(6 \mathrm{~h})$, levels of SRC-3 in gastrocnemius in wild-type animals were found to decline along with the level of GCN5 (Coste et al. 2008). Concomitant with the decrease in GCN5, the total acetylation state of PGC- $1 \alpha$ fell. When wild-type animals were fed a high-fat diet for $4 \mathrm{wk}, \mathrm{SRC}-3$ protein in gastrocnemius was elevated, as was GCN5 expression. According to the model proposed by the authors, diet
Figure 1. Illustration of the signaling pathways that regulate PGC- $1 \alpha$ through the control of GCN5 and Sirt1 activities. Pathways that promote the activity of GCN5 or Sirt1 are indicated by a green arrow, whereas those that inhibit the activity of the respective enzymes are indicated by a red arrow. See text for additional details.

composition/nutrient availability directly regulate the expression of SRC-3, which subsequently influences the expression of GCN5, which, in turn, alters the acetylation state/cotranscriptional activity of PGC- $1 \alpha$.

Angiotensin II, a hormone involved in blood pressure regulation and implicated in the pathogenesis of hypertension and type II diabetes, has been reported to increase PGC-1 $\alpha$ 's acetylation and suppress its ability to increase catalase (Xiong et al. 2010). Angiotensin II was found to activate Akt kinase, which phosphorylated PGC- $1 \alpha$ at serine 570. Phosphorylation at this site was required for the binding of GCN5 and the acetylation of PGC- $1 \alpha$ in vascular smooth muscle cells; angiotensin II was unable to induce acetylation in an S570A mutant of PGC- $1 \alpha$. Interestingly, these data would imply that other regulators of Akt activity, such as insulin, would also be able to modulate PGC- $1 \alpha$ through a similar mechanism.

Fluctuations in the availability of the GCN5 cosubstrate acetyl-CoA is another conceivable mechanism by which PGC- $1 \alpha$ acetylation can be controlled. Changes in the levels of acetyl-CoA would directly impact the reaction rate of GCN5. One of the determinants of the nuclear pool of acetyl-CoA is glucose-derived citrate, which is used by the enzyme ATP citrate lyase (ACL) to produce acetyl-CoA in both the cytoplasm and nucleus. Knockdown of ACL has been shown to globally reduce histone acetylation, suppress the expression of several genes associated with carbohydrate metabolism, and prevent the induction of GLUT4 by glucose in the 3T3-L1 cell line (Wellen et al. 2009). Whether any of these metabolic changes are directly due to GCN5's effects on PGC- $1 \alpha$ has yet to be determined.

\section{The Relationship between Sirt1 and PGC-1 $\alpha$ Acetylation and its Regulation by Nutrient Supply and Hormones}

Sirt1 has thus far been the only identified protein capable of binding to PGC- $1 \alpha$ and deacetylating it both in vivo and in vitro (Nemoto et al. 2005; Rodgers et al. 2005). Sirt1 binds to a region of PGC- $1 \alpha$ that is contained within amino acid residues 200-400 and deacetylates the protein in an $\mathrm{NAD}^{+}$-dependent manner (Nemoto et al. 2005; Rodgers et al. 2005). Changes in the level of 
Sirt1 activity are associated with changes in the expression of PGC- $1 \alpha$ targets in liver and muscle tissue. In hepatoma cultures, knockdown of Sirt1 significantly increased the acetylation of PGC- $1 \alpha$ and reduced both the induction of gluconeogenic genes and the rate at which glucose was produced following PGC-1 $\alpha$ overexpression (Rodgers et al. 2005). In myotube cultures, Sirt1 overexpression significantly decreased PGC- $1 \alpha$ acetylation and increased the expression of PGC-1 $\alpha$ targets; knockdown, on the other hand, produced a corresponding decrease in the expression of PGC- $1 \alpha$ targets. Activation of endogenous Sirt1 activity by resveratrol in cultures of C2C12 myotubes also induced the deacetylation of overexpressed PGC- $1 \alpha$ protein and potentiated the effects of PGC- $1 \alpha$ on MCAD, ERR $\alpha$, and cytochrome C (Lagouge et al. 2006).

In agreement with the cell culture studies, overexpression of Sirt1 in the liver significantly increased the expression of PEPCK and G-6-Pase in a PGC- $1 \alpha$-dependent manner (Rodgers and Puigserver 2007). Conversely, knockdown of Sirt1 attenuated hepatic glucose output induced by PGC- $1 \alpha$ overexpression in a pyruvate tolerance test in mice (Rodgers and Puigserver 2007) and reduced both fasting glucose levels and hepatic gluconeogenesis in rats (Erion et al. 2009). Experimental evidence suggests that there are three principal mechanisms by which Sirt1 activity is modulated in cells and, therefore, affects the acetylation of PGC- $1 \alpha$. These mechanisms are (i) regulation of protein expression, (ii) regulation of catalytic activity by changes in $\mathrm{NAD}^{+}$levels, and (iii) regulation of catalytic activity by posttranslation modification and binding partner interaction. Levels of Sirt1 have been reported to change in response to nutrients. Some investigators have found that nutrient restriction increases Sirt1 transcription by a mechanism that depends on either the transcription factor Foxo3a (Nemoto et al. 2004) or the transcriptional corepressor CtBP and its binding partner Hypermethylated In Cancer (Zhang et al. 2007). Low nutrient availability coupled with an elevation in catecholamines has also been reported to activate Sirt1 transcription through activation of cAMP response element binding protein (Noriega et al. 2011). High nutrient availability, in contrast, has been shown to result in active suppression of Sirt1 gene transcription by ChREBP (Noriega et al. 2011). Others, however, report that there are increases in Sirt1 protein caused by nutrient deprivation in both cultured cells and mice, but they are not accompanied by changes in the rates of transcription or in steady-state mRNA levels (Rodgers et al. 2005; Kanfi et al. 2008).

$\mathrm{NAD}^{+}$is a necessary cosubstrate for the deacetylase activity of sirtuin proteins. Cell culture experiments in which $\mathrm{NAD}^{+}$biosynthesis was manipulated by varying the expression level of nicotinamide phosphoribosyl transferase (NAMPT) — an enzyme involved in the salvaging of $\mathrm{NAD}^{+}$from nicotinamide - have shown that it is possible to produce significant changes in Sirt1 activity by altering $\mathrm{NAD}^{+}$levels (Revollo et al. 2004). Furthermore, there is evidence that $\mathrm{NAD}^{+}$levels change in response to physiological stimuli, such as nutrient availability and hormonal signaling cascades. Specifically, intracellular concentrations of liver and muscle $\mathrm{NAD}^{+}$have been shown to be increased in rodents during fasting (Rodgers et al. 2005; Yang et al. 2007; Canto et al. 2009). It is not clear, however, if these changes are in response to a particular macronutrient such as glucose or fatty acids, a downstream metabolite, or a fasting hormonal signal. The observation that cultured cells show an increase in intracellular $\mathrm{NAD}^{+}$following the withdrawal of glucose in culture medium alone (Rodgers et al. 2005; Gerhart-Hines et al. 2007) suggests that it is possible that $\mathrm{NAD}^{+}$levels within tissues in vivo are also changing, at least in part, upon glucose availability. Research with cell culture models has led to a model proposing that reduced glucose availability causes a decrease in the synthesis of ATP and an increase in AMP levels, which facilitates activation of AMPK. The activation of AMPK, in turn, produces an increase in the rate of $\mathrm{NAD}^{+}$biosynthesis and the resulting elevation in $\mathrm{NAD}^{+}$yields a wholesale increase in sirtuin activity. There have been two different proposals for how changes in AMPK activity are eventually translated into an increase in $\mathrm{NAD}^{+}$biosynthesis. One proposal is that activation of AMPK causes an increase in the expression of NAMPT and increases the rate at which $\mathrm{NAD}^{+}$is synthesized from nicotinamideindeed, knockdown of NAMPT was able to prevent $\mathrm{NAD}^{+}$levels from rising in $\mathrm{C} 2 \mathrm{C} 12$ skeletal muscle cells cultured in low-glucose medium (Fulco et al. 2008). Another proposal suggests that AMPK activation produces changes in $\mathrm{NAD}^{+}$independently of changes in NAMPT levels. C2C12 cells cultured under low-glucose conditions were found to have elevated AMPK activity and $\mathrm{NAD}^{+}$levels but no change in NAMPT expression (Canto et al. 2009). The significant delay between the onset of AMPK activation and the point at which intracellular $\mathrm{NAD}^{+}$levels were observed to change suggested that some as yet unidentified intermediary response may be involved in initiating the change in $\mathrm{NAD}^{+}$. Regardless of the mechanism by which AMPK increases $\mathrm{NAD}^{+}$levels, whole animal models have reinforced the idea that conditions that activate AMPK induce Sirtl activity through changes in $\mathrm{NAD}^{+}$. Intense exercise, which causes AMPK activation in skeletal muscle, is associated with an increase in $\mathrm{NAD}^{+}$levels, a decrease in PGC- $1 \alpha$ acetylation, and an increase in PGC- $1 \alpha$ target expression (Canto et al. 2009; although see Philp et al. 2011). Hormonal signals have also been shown to modulate this pathway. Adiponectin, through the intermediacy of AMPK, was shown to induce $\mathrm{NAD}^{+}$levels in myocytes and cause PGC-1 $\alpha$ deacetylation (Iwabu et al. 2010). FGF21 has also been demonstrated to cause AMPK activation and PGC-1 $\alpha$ deacetylation in adipocytes (Chau et al. 2010).

There are two major posttranslational mechanisms by which Sirt1 activity can be physiologically regulated. The first mechanism is by protein phosphorylation. The second is by interactions with accessory proteins that either promote or suppress the catalytic activity of the enzyme. Phosphorylation-mediated activation of Sirt1 has previously been reported for the kinases DYRK1A and 
DYRK3 (Guo et al. 2010), JNK1 (Nasrin et al. 2009), and CK2 (Kang et al. 2009; Zschoernig and Mahlknecht 2009). Because the sites of phosphorylation lie outside of the catalytic domain, the mechanism by which catalytic activation is achieved is not clear. Because PGC- $1 \alpha$ acetylation/activity was not assessed in these studies, it is also not clear as to what extent they affect the metabolic pathways associated with PGC- $1 \alpha$.

Our laboratory has recently identified a conserved phosphorylation site (Ser434 in mouse Sirt1) in the catalytic domain of Sirt1 that is phosphorylated upon activation of the PKA pathway (Gerhart-Hines et al. 2011). Structurally, Ser434 is located at the edge of the NAD ${ }^{+}$ binding pocket of Sirt1 and, together with Arg266, forms a clasp that helps encapsulate the disphosphate backbone of $\mathrm{NAD}^{+}$. Sirt1 protein immunoprecipitated from cells treated with PKA agonists showed a very rapid phosphorylation of Ser434 that corresponded to a decrease in PGC$1 \alpha$ acetylation. Similarly, animals exposed to either PKA agonists or an acute cold challenge also showed an increase in Ser434 phosphorylation. An analysis of Sirt1's enzyme kinetics revealed that phosphorylation in response to PKA activation significantly reduced the $K_{\mathrm{m}}$ for $\mathrm{NAD}^{+}$and increased the overall reaction velocity. These changes in kinetics could be blocked in nonphosphorylatable mutants (Ser434Ala and Ser434Cys). Metabolically, it was found that phosphorylation of Ser434 was necessary to see the full induction of fatty acid oxidation that is normally seen in response to PKA stimulation. Many of the transcriptional responses in PGC- $1 \alpha-$ targeted metabolic genes that are normally induced upon PKA stimulation, such as PDK4 and IDH3a, also depended on phosphorylation at Ser434. Overall, these data indicate that the regulated phosphorylation of Sirt1 at Ser434 by the PKA pathway plays an important role in coordinating the deacetylation of PGC-1 $\alpha$ and the activation of fatty acid-utilization pathways.

There are two proteins that have been reported to modulate the activity of Sirt1: Active Regulator of Sirt1 (AROS) and Deleted in Breast Cancer-1 (DBC-1). AROS protein increases Sirt1 activity (Kim et al. 2007), whereas DBC-1 decreases the activity of Sirt1 (Kim et al. 2008; Zhao et al. 2008). Despite their capacity to regulate Sirt1 activity, little is known about whether these proteins are also able to affect PGC- $1 \alpha$ acetylation.

\section{CONCLUSIONS}

Research into the control of PGC- $1 \alpha$ acetylation has identified the enzymes GCN5 and Sirt1 as important contributors to the acetylation of this powerful transcriptional coactivator. A major research prerogative now is to identify all of the pathways that converge upon GCN5 and Sirt1 to regulate their activity. Recent studies, which have unearthed only a handful of pathways, have most likely only scratched the surface of an otherwise very complicated array of signaling cascades that are involved in the maintenance of mammalian metabolic homeostasis. The delineation of these pathways will permit a new foothold for the development of therapies for the treatment of disorders with a dysfunction of central metabolism, such as obesity and type II diabetes.

\section{REFERENCES}

Badman MK, Koester A, Flier JS, Kharitonenkov A, MaratosFlier E. 2009. Fibroblast growth factor 21-deficient mice demonstrate impaired adaptation to ketosis. Endocrinology 150: $4931-4940$

Brown K, Chen Y, Underhill TM, Mymryk JS, Torchia J. 2003. The coactivator $\mathrm{p} / \mathrm{CIP} / \mathrm{SRC}-3$ facilitates retinoic acid receptor signaling via recruitment of GCN5. J Biol Chem 278: 39402-39412.

Cannon B, Nedergaard J. 2004. Brown adipose tissue: Function and physiological significance. Physiol Rev 84: 277-359.

Canto C, Gerhart-Hines Z, Feige JN, Lagouge M, Noriega L, Milne JC, Elliott PJ, Puigserver P, Auwerx J. 2009. AMPK regulates energy expenditure by modulating $\mathrm{NAD}^{+}$metabolism and SIRT1 activity. Nature 458: 1056-1060.

Chau MD, Gao J, Yang Q, Wu Z, Gromada J. 2010. Fibroblast growth factor 21 regulates energy metabolism by activating the AMPK-SIRT1-PGC-1 $\alpha$ pathway. Proc Natl Acad Sci 107: $12553-12558$.

Coste A, Louet JF, Lagouge M, Lerin C, Antal MC, Meziane H, Schoonjans K, Puigserver P, O'Malley BW, Auwerx J. 2008. The genetic ablation of SRC-3 protects against obesity and improves insulin sensitivity by reducing the acetylation of PGC-1 $\alpha$. Proc Natl Acad Sci 105: 17187-17192.

Cunningham JT, Rodgers JT, Arlow DH, Vazquez F, Mootha VK, Puigserver P. 2007. mTOR controls mitochondrial oxidative function through a YY1-PGC-1 $\alpha$ transcriptional complex. Nature 450: 736-740.

Erion DM, Yonemitsu S, Nie Y, Nagai Y, Gillum MP, Hsiao JJ, Iwasaki T, Stark R, Weismann D, Yu XX, et al. 2009. SirT1 knockdown in liver decreases basal hepatic glucose production and increases hepatic insulin responsiveness in diabetic rats. Proc Natl Acad Sci 106: 11288-11293.

Fulco M, Cen Y, Zhao P, Hoffman EP, McBurney MW, Sauve AA, Sartorelli V. 2008. Glucose restriction inhibits skeletal myoblast differentiation by activating SIRT1 through AMPKmediated regulation of Nampt. Dev Cell 14: 661-673.

Gerhart-Hines Z, Rodgers JT, Bare O, Lerin C, Kim SH, Mostoslavsky R, Alt FW, Wu Z, Puigserver P. 2007. Metabolic control of muscle mitochondrial function and fatty acid oxidation through SIRT1/PGC-1 $\alpha$. EMBO J 26: 19131923.

Gerhart-Hines Z, Dominy JE Jr, Blattler SM, Jedrychowski MP, Banks AS, Lim JH, Chim H, Gygi SP, Puigserver P. 2011. The cAMP/PKA pathway rapidly activates SIRT1 to promote fatty acid oxidation independently of changes in $\mathrm{NAD}(+) . \mathrm{Mol}$ Cell 44: 851-863.

Guo X, Williams JG, Schug TT, Li X. 2010. DYRK1A and DYRK3 promote cell survival through phosphorylation and activation of SIRT1. J Biol Chem 285: 13223-13232.

Hardie DG. 2007. AMP-activated/SNF1 protein kinases: Conserved guardians of cellular energy. Nat Rev Mol Cell Biol 8: 774-785.

Inagaki T, Dutchak P, Zhao G, Ding X, Gautron L, Parameswara V, Li Y, Goetz R, Mohammadi M, Esser V, et al. 2007. Endocrine regulation of the fasting response by PPAR $\alpha$-mediated induction of fibroblast growth factor 21. Cell Metab 5: $415-425$.

Iwabu M, Yamauchi T, Okada-Iwabu M, Sato K, Nakagawa T, Funata M, Yamaguchi M, Namiki S, Nakayama R, Tabata M, et al. 2010. Adiponectin and AdipoR1 regulate PGC-1 $\alpha$ and mitochondria by $\mathrm{Ca}(2+)$ and AMPK/SIRT1. Nature 464: 1313-1319.

Jacob F, Perrin D, Sanchez C, Monod J. 1960. [Operon: A group of genes with the expression coordinated by an 
operator] (article in French). CR Hebd Seances Acad Sci 250: 1727-1729.

Kahn BB, Alquier T, Carling D, Hardie DG. 2005. AMP-activated protein kinase: Ancient energy gauge provides clues to modern understanding of metabolism. Cell Metab 1: 15-25.

Kanfi Y, Peshti V, Gozlan YM, Rathaus M, Gil R, Cohen HY. 2008. Regulation of SIRT1 protein levels by nutrient availability. FEBS Lett 582: 2417-2423.

Kang H, Jung JW, Kim MK, Chung JH. 2009. CK2 is the regulator of SIRT1 substrate-binding affinity, deacetylase activity and cellular response to DNA-damage. PloS One 4: e6611.

Kelly DP, Scarpulla RC. 2004. Transcriptional regulatory circuits controlling mitochondrial biogenesis and function. Genes Dev 18: 357-368.

Kim EJ, Kho JH, Kang MR, Um SJ. 2007. Active regulator of SIRT1 cooperates with SIRT1 and facilitates suppression of p53 activity. Mol Cell 28: 277-290.

Kim JE, Chen J, Lou Z. 2008. DBC1 is a negative regulator of SIRT1. Nature 451: 583-586.

Lagouge M, Argmann C, Gerhart-Hines Z, Meziane H, Lerin C, Daussin F, Messadeq N, Milne J, Lambert P, Elliott P, et al. 2006. Resveratrol improves mitochondrial function and protects against metabolic disease by activating SIRT1 and PGC-1 $\alpha$. Cell 127: 1109-1122.

Lerin C, Rodgers JT, Kalume DE, Kim SH, Pandey A, Puigserver P. 2006. GCN5 acetyltransferase complex controls glucose metabolism through transcriptional repression of PGC1 $\alpha$. Cell Metab 3: 429-438.

Lin J, Wu PH, Tarr PT, Lindenberg KS, St-Pierre J, Zhang CY, Mootha VK, Jager S, Vianna CR, Reznick RM, et al. 2004. Defects in adaptive energy metabolism with CNS-linked hyperactivity in PGC-1 $\alpha$ null mice. Cell 119: $121-135$.

Mayr B, Montminy M. 2001. Transcriptional regulation by the phosphorylation-dependent factor CREB. Nat Rev Mol Cell Biol 2: 599-609.

Mootha VK, Lindgren CM, Eriksson KF, Subramanian A, Sihag S, Lehar J, Puigserver P, Carlsson E, Ridderstrale M, Laurila $\mathrm{E}$, et al. 2003. PGC-1 $\alpha$-responsive genes involved in oxidative phosphorylation are coordinately downregulated in human diabetes. Nat Genet 34: 267-273.

Nasrin N, Kaushik VK, Fortier E, Wall D, Pearson KJ, de Cabo R, Bordone L. 2009. JNK1 phosphorylates SIRT1 and promotes its enzymatic activity. PloS one 4: e8414.

Nemoto S, Fergusson MM, Finkel T. 2004. Nutrient availability regulates SIRT1 through a forkhead-dependent pathway. Science 306: 2105-2108.

Nemoto S, Fergusson MM, Finkel T. 2005. SIRT1 functionally interacts with the metabolic regulator and transcriptional coactivator PGC-1 $\alpha$. J Biol Chem 280: 16456-16460.

Noriega LG, Feige JN, Canto C, Yamamoto H, Yu J, Herman MA, Mataki C, Kahn BB, Auwerx J. 2011. CREB and
ChREBP oppositely regulate SIRT1 expression in response to energy availability. EMBO Rep 12: 1069-1076.

Philp A, Chen A, Lan D, Meyer GA, Murphy AN, Knapp AE, Olfert IM, McCurdy CE, Marcotte GR, Hogan MC, et al. 2011. Sirtuin 1 (SIRT1) deacetylase activity is not required for mitochondrial biogenesis or peroxisome proliferatoractivated receptor- $\gamma$ coactivator- $1 \alpha$ (PGC-1 $\alpha$ ) deacetylation following endurance exercise. J Biol Chem 286: $30561-$ 30570 .

Puigserver P, Spiegelman BM. 2003. Peroxisome proliferatoractivated receptor- $\gamma$ coactivator $1 \alpha$ (PGC- $1 \alpha)$ : Transcriptional coactivator and metabolic regulator. Endocr Rev 24: 7890.

Rasbach KA, Gupta RK, Ruas JL, Wu J, Naseri E, Estall JL, Spiegelman BM. 2010. PGC- $1 \alpha$ regulates a HIF $2 \alpha$-dependent switch in skeletal muscle fiber types. Proc Natl Acad Sci 107: 21866-21871.

Revollo JR, Grimm AA, Imai S. 2004. The NAD biosynthesis pathway mediated by nicotinamide phosphoribosyltransferase regulates Sir2 activity in mammalian cells. J Biol Chem 279: 50754-50763.

Rodgers JT, Puigserver P. 2007. Fasting-dependent glucose and lipid metabolic response through hepatic sirtuin 1. Proc Natl Acad Sci 104: 12861-12866.

Rodgers JT, Lerin C, Haas W, Gygi SP, Spiegelman BM, Puigserver P. 2005. Nutrient control of glucose homeostasis through a complex of PGC- $1 \alpha$ and SIRT1. Nature 434: $113-$ 118.

Wellen KE, Hatzivassiliou G, Sachdeva UM, Bui TV, Cross JR, Thompson CB. 2009. ATP-citrate lyase links cellular metabolism to histone acetylation. Science 324: 1076-1080.

Xiong S, Salazar G, San Martin A, Ahmad M, Patrushev N, Hilenski L, Nazarewicz RR, Ma M, Ushio-Fukai M, Alexander RW. 2010. PGC-1 $\alpha$ serine 570 phosphorylation and GCN5-mediated acetylation by angiotensin II drive catalase down-regulation and vascular hypertrophy. J Biol Chem 285: 2474-2487.

Yang H, Yang T, Baur JA, Perez E, Matsui T, Carmona JJ, Lamming DW, Souza-Pinto NC, Bohr VA, Rosenzweig A, et al. 2007. Nutrient-sensitive mitochondrial $\mathrm{NAD}^{+}$levels dictate cell survival. Cell 130: 1095-1107.

Zhang Q, Wang SY, Fleuriel C, Leprince D, Rocheleau JV, Piston DW, Goodman RH. 2007. Metabolic regulation of SIRT1 transcription via a HIC1:CtBP corepressor complex. Proc Natl Acad Sci 104: 829-833.

Zhao W, Kruse JP, Tang Y, Jung SY, Qin J, Gu W. 2008. Negative regulation of the deacetylase SIRT1 by DBC1. Nature 451: $587-590$.

Zschoernig B, Mahlknecht U. 2009. Carboxy-terminal phosphorylation of SIRT1 by protein kinase CK2. Biochem Biophys Res Commun 381: 372-377. 


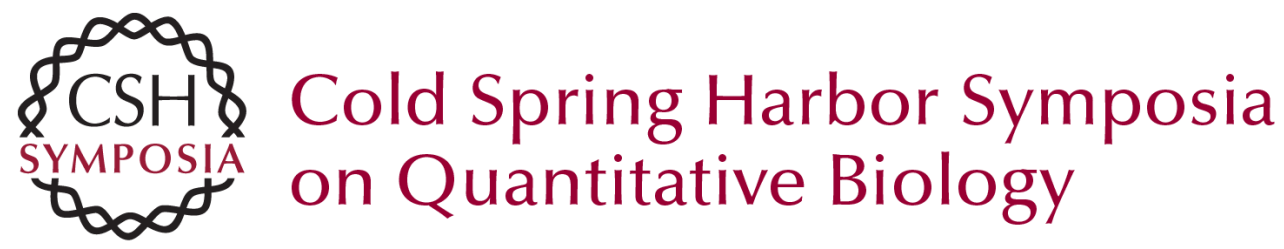

\section{Nutrient-Dependent Acetylation Controls Basic Regulatory Metabolic Switches and Cellular Reprogramming}

J.E. Dominy, Z. Gerhart-Hines and P. Puigserver

Cold Spring Harb Symp Quant Biol 2011 76: 203-209 originally published online February 27, 2012

Access the most recent version at doi:10.1101/sqb.2012.76.010843

References This article cites 43 articles, 15 of which can be accessed free at: http://symposium.cshlp.org/content/76/203.full.html\#ref-list-1

\section{License}

Email Alerting Service the box at the top right corner of the article or click here. 